\title{
Lattice Boltzmann simulations of segregating binary fluid mixtures in shear flow
}

\author{
A. Lamura ${ }^{1,2}$ and G. Gonnella ${ }^{1}$ \\ ${ }^{1}$ Istituto Nazionale per la Fisica della Materia, Unità di Bari and \\ Dipartimento di Fisica dell'Università di Bari and \\ Istituto Nazionale di Fisica Nucleare, Sezione di Bari \\ via Amendola 173, 70126 Bari, Italy \\ ${ }^{2}$ Institut für Festkörperforschung, Forschungszentrum Jülich, \\ 52425 Jülich, Germany
}

\begin{abstract}
We apply lattice Boltzmann method to study the phase separation of a two-dimensional binary fluid mixture in shear flow. The algorithm can simulate systems described by the Navier-Stokes and convection-diffusion equations. We propose a new scheme for imposing the shear flow which has the advantage of preserving mass and momentum conservation on the boundary walls without introducing slip velocities. Our main results concern the presence of two typical lenght scales in the phase separation process, corresponding to domains with two different thicknesses. Our simulations at low viscosity confirm previous results only valid in the limit of infinite viscosity.
\end{abstract}




\section{Introduction}

When a fluid mixture is suddenly quenched from a disordered initial condition to a coexistence state below the critical temperature, the two fluids segregate with domains growing with time (see, e.g., Gunton et al. 1983). The morphology of these domains is strongly influenced by an applied flow. In the case of a shear flow (for a review see Onuki 1997), the domains are observed to grow greatly elongated in the flow direction (see, e.g., Hashimoto et al. 1995). The effects of the shear have been considerably investigated in the last years. Several numerical simulations confirm the anisotropic growth of the domains (Ohta et al. 1990, Rothman 1991, Olson \& Rothman 1995, Wu et al. 1997, Padilla \& Toxvaerd 1997, Shou \& Chakrabarti 2000). New recent theoretical results by Corberi et al. (1998)-(1999) show that, as an effect of the shear, the mixture contains domains of two different thicknesses and that the relative abundance of these domains changes periodically with the logarithm of time. However in the papers of Corberi et al. (1998)-(1999) phase separation occurred only for the effects of simple diffusion. The main purpose of this paper is to see how the hydrodynamics affects this picture.

The presence of domains with different scales is a very peculiar result for phase separating mixtures. Indeed, in the general picture for the unsheared case, a single time-dependent length scale $R(t)$, which measures the typical size of domains, characterizes the behaviour of the mixture (see Bray 1994). This length grows with the power law $R(t) \sim t^{\alpha}$, where the value of the growth exponent $\alpha$ is strictly related to the physical mechanism operating

in the segregation process. For example $\alpha=1 / 3$ in a pure diffusive regime. Therefore the existence of two characteristic scales makes the phase separation process very interesting 
from a theoretical point of view and also with relevant practical implications.

Looking at the effects of hydrodynamics on this problem is not a simple computational task. Domains grow fast in the direction of the flow making finite-size effects quite soon relevant in simulations (for a recent review, see, Yeomans 1999). In this paper we use a lattice Boltzmann scheme (Orlandini et al. 1995, Swift et al. 1996) to simulate the convection-diffusion and Navier-Stokes equations for a binary mixture. This method has been found very convenient with respect to other numerical algorithms for fluid mixtures (Chen \& Doolen 1998) since it allows to reach very large time scales. In particular, an advantage of the lattice Boltzmann scheme of Orlandini et al. (1995) and Swift et al. (1996), is that the correct fluid equilibrium can be imposed by choosing an appropriate free energy. Moreover, differently from other lattice gas methods, it allows to control in a separate way the different mechanisms of transport tuning independently the fluid viscosity and the diffusivity. The mentioned lattice Boltmann scheme has been used with success in several problems of phase separation of binary fluid mixtures without shear in two (Osborn et al. 1995) and three dimensions (Kendon et al. 1999), and also in the case of complex fluids (Gonnella et al. 1997).

A preliminary important part of our work has been to include proper boundary conditions for a shear flow in lattice Boltzmann schemes. This problem, considered also by Wagner \& Yeomans (1998) and Cates et al. (1999), has been studied in many papers on lattice Boltzmann schemes for a single fluid (for a review, see, e. g., Chen \& Doolen 1998). We discuss previous procedures and propose an improved algorithm which allows to strictly conserve mass and momentum on the boundary walls without introducing slip velocities. 
The outline of the paper is as follows. In the next section we describe the fluid model and the lattice Boltzmann scheme. In section 3 we describe and compare different approaches for implementing shear boundary conditions in the numerical scheme. In section 4 we give our results for the phase separation and finally we draw our conclusions.

\section{The lattice Boltzmann method}

Our simulations are based on a lattice Boltzmann scheme developed by Orlandini et al. (1995) and Swift et al. (1996). In this scheme the choice of a free energy determines all the thermodinamic properties of the fluid. We start from the description of the free energy of the fluid mixture; then we show how the lattice Boltzmann equations have been implemented.

Equilibrium description of the mixture. The free-energy functional generally used in phase separation studies of binary mixtures (Bray 1994) is

$$
\mathcal{F}=\int d \mathbf{r}\left[\frac{a}{2} \varphi^{2}+\frac{b}{4} \varphi^{4}+\frac{\kappa}{2}(\nabla \varphi)^{2}\right]
$$

where $\varphi$ is the order parameter which describes the normalized difference in the densities of the two fluids. The polynomial terms are related to the bulk properties of the fluid. While the parameter $b$ is always positive, the sign of $a$ distinguishes between a disordered and a segregated mixture. The case with $a>0$ gives a polynomial with one minimum in the origin corresponding to the a state with $\varphi=0$ everywhere while two minima are present when $a<0$ corresponding to the two pure phases with $\varphi= \pm \sqrt{-a / b}$. In the following we will consider a deep quench (well below the critical value $a=0$ ) into the coexistence region with $a=-1$ and $b=1$. The gradient term is related to the interfacial properties. The equilibrium

profile between the two coexisting bulk phases is $\varphi(x)=\tanh \sqrt{\frac{1}{2 \kappa}} x$ giving a surface tension 
equal to $\frac{2}{3} \sqrt{2 \kappa}$ and an interfacial width proportional to $\sqrt{2 \kappa}$ (Rowlinson \& Widom 1982). The total fluid density $n$ is not explicitly included in (1) because we are dealing with an incompressible mixture with $n$ constant throughout the volume of the fluid and throughout its motion.

The thermodynamic properties of the fluid follow from the free energy (1) (Reichl 1980). The chemical potential difference between the two fluids is given by

$$
\Delta \mu=\frac{\delta \mathcal{F}}{\delta \varphi}=a \varphi+b \varphi^{3}-\kappa \nabla^{2} \varphi
$$

The pressure is not a scalar but a tensor $P_{\alpha \beta}$ since interfaces in the fluid can exert nonisotropic forces (Yang et al. 1976). The scalar part $p_{o}$ follows directly from (11)

$$
\begin{aligned}
p_{o} & =\varphi \frac{\delta \mathcal{F}}{\delta \varphi}-f(\varphi) \\
& =\frac{a}{2} \varphi^{2}+\frac{3 b}{4} \varphi^{4}-\kappa \varphi\left(\nabla^{2} \varphi\right)-\frac{\kappa}{2}(\nabla \varphi)^{2}
\end{aligned}
$$

where $f(\varphi)$ is the free-energy density. In order to calculate the pressure tensor $P_{\alpha \beta}$, one has to ensure that $P_{\alpha \beta}$ obeys the condition of mechanical equilibrium $\partial_{\alpha} P_{\alpha \beta}=0$ (Evans 1979). A suitable choice is

$$
P_{\alpha \beta}=p_{o} \delta_{\alpha \beta}+\kappa \partial_{\alpha} \varphi \partial_{\beta} \varphi
$$

Lattice Boltzmann scheme. We use a square lattice in which each site has eight nearest neighbours. The lattice has horizontal and vertical links of unit length and diagonal links of length $\sqrt{2}$. We denote by $L$ the number of lattice sites in each direction. The variables of the lattice Boltzmann algorithm are two sets of distribution functions $f_{i}(\mathbf{r}, t)$ and $g_{i}(\mathbf{r}, t)$, defined on each lattice site $\mathbf{r}$ at time $t$. Each of them is associated with a velocity vector $\mathbf{e}_{i}$. Defined $\Delta t$ as the simulation time step, the quantities $\mathbf{e}_{i} \Delta t$ are constrained to be lattice 
vectors: $\left|\mathbf{e}_{i}\right| \Delta t=1$ for the horizontal and vertical directions $(i=1,2,3,4)$ and $\left|\mathbf{e}_{i}\right| \Delta t=\sqrt{2}$ for the diagonal directions $(i=5,6,7,8)$. In figure 1 we show a plaquette of the lattice with the velocity vectors. Two functions $f_{0}(\mathbf{r}, t)$ and $g_{0}(\mathbf{r}, t)$, corresponding to the distribution components that do not propagate $\left(\mathbf{e}_{0}=\mathbf{0}\right)$, are also taken into account.

The distribution functions are related to the total density $n$, to the fluid velocity $\mathbf{u}$ and to the density difference $\varphi$ through

$$
n=\sum_{i} f_{i}, \quad n \mathbf{u}=\sum_{i} f_{i} \mathbf{e}_{i}, \quad \varphi=\sum_{i} g_{i} .
$$

They evolve during the time step $\Delta t$ according to a single relaxation-time Boltzmann equation (Bhatnagar et al. 1954, Chen et al. 1992):

$$
\begin{aligned}
& f_{i}\left(\mathbf{r}+\mathbf{e}_{i} \Delta t, t+\Delta t\right)-f_{i}(\mathbf{r}, t)=-\frac{1}{\tau}\left[f_{i}(\mathbf{r}, t)-f_{i}^{e q}(\mathbf{r}, t)\right], \\
& g_{i}\left(\mathbf{r}+\mathbf{e}_{i} \Delta t, t+\Delta t\right)-g_{i}(\mathbf{r}, t)=-\frac{1}{\tau_{\varphi}}\left[g_{i}(\mathbf{r}, t)-g_{i}^{e q}(\mathbf{r}, t)\right],
\end{aligned}
$$

where $\tau$ and $\tau_{\varphi}$ are independent relaxation parameters, $f_{i}^{e q}(\mathbf{r}, t)$ and $g_{i}^{e q}(\mathbf{r}, t)$ are local equilibrium distribution functions.

The time evolution occurs in two steps: a collision and a propagation step. Firstly, the distribution functions arriving at the same time on the same site change according to the equation (we will write it only for one of the two distributions)

$$
f_{i}^{c}(\mathbf{r}, t)=f_{i}(\mathbf{r}, t)-\frac{1}{\tau}\left[f_{i}(\mathbf{r}, t)-f_{i}^{e q}(\mathbf{r}, t)\right] .
$$

Then the distribution functions are moved along the lattice directions according to the rule

$$
f_{i}\left(\mathbf{r}+\mathbf{e}_{i} \Delta t, t+\Delta t\right)=f_{i}^{c}(\mathbf{r}, t) .
$$

Equation (6) comes from combining equations (8) and (9). 
The system is initialized with $n=1, \varphi=0$ and $\mathbf{u}=\mathbf{0}$ everywhere. The choice of a constant value of $n$ is justified by the incompressibility of the mixture. Our simulations confirm that $n$ stays constant throughout the system during all the time evolution. The initial value of $\varphi$ corresponds to a symmetric 50:50 mixture with the two fluids completely mixed. These quantities are locally conserved in any collision process and, therefore, we require that the local equilibrium distribution functions fulfil the equations

$$
\begin{aligned}
\sum_{i}\left(f_{i}^{e q}-f_{i}\right)=0 & \Rightarrow \sum_{i} f_{i}^{e q}=n \\
\sum_{i}\left(g_{i}^{e q}-g_{i}\right)=0 & \Rightarrow \sum_{i} g_{i}^{e q}=\varphi \\
\sum_{i}\left(f_{i}^{e q}-f_{i}\right) \mathbf{e}_{i}=\mathbf{0} & \Rightarrow \sum_{i} f_{i}^{e q} \mathbf{e}_{i}=n \mathbf{u}
\end{aligned}
$$

Following Orlandini et al. (1995) and Swift et al. (1996) the higher moments of the local equilibrium distribution functions are defined so that we can obtain continuum equations pertinent to a binary fluid mixture. We define

$$
\begin{gathered}
\sum_{i} f_{i}^{e q} e_{i \alpha} e_{i \beta}=P_{\alpha \beta}+n u_{\alpha} u_{\beta}, \\
\sum_{i} g_{i}^{e q} e_{i \alpha}=\varphi u_{\alpha}, \\
\sum_{i} g_{i}^{e q} e_{i \alpha} e_{i \beta}=\Gamma \Delta \mu \delta_{\alpha \beta}+\varphi u_{\alpha} u_{\beta} .
\end{gathered}
$$

where $\Gamma$ is a coefficient related to the mobility of the fluid. We are considering a mixture with the two fluids having the same mechanical properties. The constraint (12) expresses the fact that the two fluids have the same velocity. The local equilibrium distribution functions can be expressed as an expansion in terms of the velocity $\mathbf{u}$ (Orlandini et al. 1995, Swift et al. 1996):

$$
f_{0}^{e q}=A_{0}+C_{0} u^{2}
$$




$$
\begin{aligned}
& f_{i}^{e q}=A_{I}+B_{I} u_{\alpha} e_{i \alpha}+C_{I} u^{2}+D_{I} u_{\alpha} u_{\beta} e_{i \alpha} e_{i \beta}+G_{I, \alpha \beta} e_{i \alpha} e_{i \beta} \quad i=1,2,3,4 \\
& f_{i}^{e q}=A_{I I}+B_{I I} u_{\alpha} e_{i \alpha}+C_{I I} u^{2}+D_{I I} u_{\alpha} u_{\beta} e_{i \alpha} e_{i \beta}+G_{I I, \alpha \beta} e_{i \alpha} e_{i \beta} \quad i=5,6,7,8
\end{aligned}
$$

Similarly

$$
\begin{aligned}
& g_{0}^{e q}=H_{0}+J_{0} u^{2} \\
& g_{i}^{e q}=H_{I}+K_{I} u_{\alpha} e_{i \alpha}+J_{I} u^{2}+Q_{I} u_{\alpha} u_{\beta} e_{i \alpha} e_{i \beta} \quad i=1,2,3,4 \\
& g_{i}^{e q}=H_{I I}+K_{I I} u_{\alpha} e_{i \alpha}+J_{I I} u^{2}+Q_{I I} u_{\alpha} u_{\beta} e_{i \alpha} e_{i \beta} \quad i=5,6,7,8
\end{aligned}
$$

The relations (10)-(13) can be used to fix the coefficients of these expansions. The results are reported in the Appendix.

Continuum equations. It has been shown by Orlandini et al. (1995) and Swift et al. (1996) that the above described lattice Boltzmann scheme simulates the continuity, the incompressible Navier-Stokes and the convection-diffusion equations

$$
\begin{aligned}
\partial_{\alpha} u_{\alpha} & =0 \\
\partial_{t} u_{\alpha}+u_{\beta} \partial_{\beta} u_{\alpha} & =-\frac{1}{n} \partial_{\alpha} p_{o}+\nu \nabla^{2} u_{\alpha} \\
\partial_{t} \varphi+\partial_{\alpha}\left(\varphi u_{\alpha}\right) & =\Gamma \Theta \nabla^{2} \frac{\delta \mathcal{F}}{\delta \varphi}
\end{aligned}
$$

A Chapman-Enskog expansion (Chapman \& Cowling 1970, Swift et al. 1996) of equations (6) and (7) to $O\left(\Delta t^{2}\right)$ allows to show that

$$
\nu=\frac{(2 \tau-1)}{6}(\Delta t), \quad \Theta=\Delta t\left(\tau_{\varphi}-\frac{1}{2}\right)
$$

We choose $\tau_{\varphi}=(1+1 / \sqrt{3}) / 2$ in order to minimize the correction terms of order $\Delta t^{3}$. Thus we are left with two free variables $\tau$ and $\Gamma$ which control the kinematic viscosity $\nu$ and the

\footnotetext{
${ }^{1}$ Actually, spurious terms appear in equations (16)-(18), whose effects are shown negligible in the papers of Orlandini et al. (1995) and Swift et al. (1996).
} 
macroscopic mobility $\Gamma \Theta$, respectively. All the simulations were run with $\kappa=1$ (this choice corresponds to an interfacial width of approximately three lattice spacings), $\Gamma \Theta=0.2$ and units in which $\Delta t=1$.

\section{Shear boundary conditions}

The next step in implementing the algorithm is to apply a shear flow on the system. We have modified previous lattice Boltzmann schemes for a single fluid where sliding walls moving in a lattice direction were used to enforce the fluid a given velocity. The flow is assumed to be directed along the $x$-axis (horizontal on the lattice). In this direction we considered periodic boundary conditions. Then departing distribution functions on outward-pointing links reenter the lattice via corresponding inward-pointing links on the opposite boundary with no constraint on the macroscopic velocity or density. The walls are on the top and the bottom of the lattice. Here one has to face two problems: the correct value of the velocity has to be imposed to the fluid, and the distribution functions pointing outward have to be managed in such a way that mass conservation for both components and momentum conservation for the bulk are always guaranteed. In the following we discuss two possible ways for introducing the sliding walls 2.

Equilibrium scheme. In the first scheme that we have considered, the boundary walls are placed on the links beyond the upper and the lower sites at a distance equal to half

\footnotetext{
${ }^{2}$ A different approach based on Lees-Edwards boundary conditions (Lees \& Edwards (1972)) has been used by Wagner \& Yeomans (1999). These boundary conditions, widely used in molecular dynamics, identify the point at $(x, y)$ with the point at $(x+\gamma L \Delta t, y+L)$, where $\gamma$ is the shear rate. Lattice Boltzmann implementation of Lees-Edwards boundary conditions requires also to change the usual definition of the local equilibrium distribution functions so that we have preferred to use the more straightforward approach with the sliding walls.
} 
lattice spacing, in the spirit of some works on shear boundary conditions in lattice Boltzmann methods for one fluid (Cornubert et al. 1991, Ladd 1994). We assign to the local equilibrium distributions at the top (t) and the bottom (b) of the lattice the values corresponding to the velocities $w_{x, t}=\gamma \frac{L-1}{2}, w_{y, t}=0, w_{x, b}=-\gamma \frac{L-1}{2}, w_{y, b}=0$, being $L-1$ the distance between the top and the bottom of the lattice (He et al. 1997). This method is expected to be accurate for relaxation parameters near unity, since when $\tau=1$ the collision process (see equation (8)) simply replaces the distribution function with the local equilibrium value. For describing the propagation step let us refer to the upper row and to the $f_{i}$ 's. After a collision, for each site of the upper row, there are three distributions $f_{5}^{c}(t), f_{2}^{c}(t), f_{6}^{c}(t)$ pointing outward the system. The propagation is implemented with the following scheme:

$$
\begin{aligned}
f_{7}\left(\mathbf{r}+\mathbf{e}_{3} \Delta t, t+\Delta t\right) & =f_{6}^{c}(\mathbf{r}, t) \\
f_{4}(\mathbf{r}, t+\Delta t) & =f_{2}^{c}(\mathbf{r}, t) \\
f_{8}\left(\mathbf{r}+\mathbf{e}_{1} \Delta t, t+\Delta t\right) & =f_{5}^{c}(\mathbf{r}, t)
\end{aligned}
$$

This choice can be justified considering reflection of particles against the boundary wall. This allows to guarantee the conservation of mass and momentum, that on each site 9 distribution functions are still defined, and that propagation between adjacent sites on the boundary occurs simultaneously to the propagation on all the inner sites, since $f_{5}^{c}(t)$ and $f_{6}^{c}(t)$ are propagated over a distance $\sqrt{2}$ in a time step and $f_{2}^{c}(t)$ over a distance equal to one. This method, however, as expected and also shown in the following, does not work well for arbitrary values of the relaxation parameters. Therefore we heve developed a second way for introducing the sliding walls. 
Collisional scheme. This second method is an improvement of a scheme proposed by Zou \& He (1997) for one-fluid systems. In this case the boundary walls are placed on the upper and lower rows of sites (see, e.g., Noble et al. 1995, Inamuro et al. 1995). We start from the propagation step, as it is realized in the original version by Zou \& He (1997), and consider again the sites at the top of the lattice. After the propagation the functions $f_{0}(t), f_{1}(t)$, $f_{5}(t), f_{2}(t), f_{6}(t)$ and $f_{3}(t)$ are known on each site of the upper row. One uses equations (5) to determine $f_{7}(t), f_{4}(t), f_{8}(t)$ and $n$. Requiring that the wall velocities $w_{x, t}=\gamma \frac{L-1}{2}$, $w_{y, t}=0$ are imposed to the fluid, we can write

$$
\begin{aligned}
f_{7}(t)+f_{4}(t)+f_{8}(t) & =n-\left[f_{0}(t)+f_{1}(t)+f_{5}(t)+f_{2}(t)+f_{6}(t)+f_{3}(t)\right] \\
f_{8}(t)-f_{7}(t) & =n \gamma \frac{L-1}{2}-\left[f_{1}(t)-f_{3}(t)+f_{5}(t)-f_{6}(t)\right] \\
f_{7}(t)+f_{4}(t)+f_{8}(t) & =f_{5}(t)+f_{2}(t)+f_{6}(t)
\end{aligned}
$$

Consistency of equations (21) gives

$$
n=f_{0}(t)+f_{1}(t)+f_{3}(t)+2\left[f_{2}(t)+f_{6}(t)+f_{5}(t)\right]
$$

The system of equations (21) reduces to two equations with three unknown variables. To close the system of equations the bounce-back rule (Lavallée et al. 1991, Cornubert et al. 1991) is adopted for the distribution functions normal to the boundary. This means that the value of $f_{4}(t)$ is fixed assigning to it the known value of its outward-pointing counterpart $f_{2}(t)$. Then one can solve the system of equations (21) finding the solutions

$$
\begin{aligned}
& f_{4}(\mathbf{r}, t)=f_{2}(\mathbf{r}, t) \\
& f_{8}(\mathbf{r}, t)=f_{6}(\mathbf{r}, t)-\frac{1}{2}\left[f_{1}(\mathbf{r}, t)-f_{3}(\mathbf{r}, t)\right]+\frac{1}{2} n \gamma \frac{L-1}{2} \\
& f_{7}(\mathbf{r}, t)=f_{5}(\mathbf{r}, t)+\frac{1}{2}\left[f_{1}(\mathbf{r}, t)-f_{3}(\mathbf{r}, t)\right]-\frac{1}{2} n \gamma \frac{L-1}{2}
\end{aligned}
$$


With this choice for the inward-pointing distributions the desidered momentum at the boundary is achieved. At this point the collision step is applied to all sites, including the boundary ones. Unfortunately this scheme does not allow to conserve exactly the total mass due to the fact that the distribution functions $f_{5}^{c}(t), f_{2}^{c}(t)$ and $f_{6}^{c}(t)$ resulting from the collision step are not accounted for any more, and this avoids the exact mass conservation (Zou \& He 1997).

We have improved the above scheme in order to overcome the mass conservation problem. We consider again the propagation step and the problem of calculating the unknown quantities $f_{7}(t), f_{4}(t)$ and $f_{8}(t)$. After the propagation step on each upper site one has the distribution functions $f_{1}(t), f_{5}(t), f_{2}(t), f_{6}(t)$ and $f_{3}(t)$ coming from the nearest sites, $f_{0}(t-\Delta t)$, which does not propagate, and $f_{5}(t-\Delta t), f_{2}(t-\Delta t)$ and $f_{6}(t-\Delta t)$, all referred to the previous time step, which were lost in the version of Zou \& He (1997). Mass will be conserved if the total density $n$ on each site is equal to the quantity $\hat{n}$ given by the sum

$$
\begin{aligned}
\hat{n}(t, t-\Delta t)= & f_{0}(t-\Delta t)+f_{5}(t-\Delta t)+f_{2}(t-\Delta t)+f_{6}(t-\Delta t) \\
+ & f_{1}(t)+f_{5}(t)+f_{2}(t)+f_{6}(t)+f_{3}(t) .
\end{aligned}
$$

We also require that equations (21) are fulfilled. In order to impose the constraint that on all the boundary sites $n=\hat{n}$, we have to introduce an extra degree of freedom in the system of equations. We have choosed $f_{0}(t)$ since it does not propagate. The solutions of the system of equations (21), $n=\hat{n}$ and (24) are

$$
f_{0}(\mathbf{r}, t)=\hat{n}-\left[f_{1}(\mathbf{r}, t)+f_{3}(\mathbf{r}, t)\right]-2\left[f_{2}(\mathbf{r}, t)+f_{5}(\mathbf{r}, t)+f_{6}(\mathbf{r}, t)\right]
$$

and again the $(23)$. In this way it is guaranteed that

$$
n(\mathbf{r}, t)=\hat{n}(\mathbf{r}, t, t-\Delta t)
$$




$$
\begin{aligned}
& n u_{x}(\mathbf{r}, t)=n \gamma \frac{L-1}{2} \\
& n u_{y}(\mathbf{r}, t)=0
\end{aligned}
$$

By this procedure, once the system has been initialized, the application of the propagation and collision steps goes on preserving mass and momentum conservation and implementing the correct velocity values on the boundaries, as it has also been verified numerically.

Comparison between the two schemes. Here we compare the validity of the two schemes. We have studied how the steady state velocity profile is reached and we have measured the slip velocity. The shear is applied as usual from the beginning of the phase separation. Preliminarly we have checked that the boundary conditions do not introduce any artificial discontinuity on the walls. Therefore we have considered the behaviour of the densities $n$ and $\varphi$ along vertical sections normal to the flow direction. In figure 2 (a)-(b) the plots of $n$ and $\varphi$, for the middle section of the system, are showed for the collisional scheme. It can be seen that the total density $n$ stays constant and equal to 1 all over the system (its deviations from 1 are less than $1 / 10^{5}$ ) and no boundary effects can be observed. The profile of $\varphi$, with the fluctuations corresponding to the presence of interfaces, does not show anything pathological, too. Similar results have been obtained with the equilibrium scheme. In figure 3 we show at consecutive times the $x$-component of the fluid velocity along a vertical line in the middle of the system calculated with the collisional scheme. The velocity profiles are independent on the particular vertical section considered. The steady velocity profile is the planar Couette flow (Landau \& Lifshitz 1959) with the $x$-component of the velocity having a linear dependence on the $y$ coordinate. The evolution of the velocity profile in figure 3 is very similar to that observed in simple fluids (Schlichting 1979, p. 92). The same evolution 
is obtained with the equilibrium scheme when $\tau=1$. The situation is different for larger values of $\tau$, when, with the first scheme, the time needed to reach the steady velocity profile is much longer. Actually, it happens that for large enough values of $\tau$ the linear profile is not reached before finite-size effects become relevant in the simulations. This can be understood from equation (\$). Indeed, if $\tau>1, f_{i}^{c}(\mathbf{r}, t)$ takes more time to reach the local equilibrium value $f_{i}^{e q}(\mathbf{r}, t)$ which contains the information about the velocity of walls.

A measure of the time $t_{r}$ required to reach the steady state velocity profile can be done in this way. We say that the steady state is reached if

$$
\frac{\sum_{\mathbf{r}}\left|u_{x}(\mathbf{r}, t+\Delta t)-u_{x}(\mathbf{r}, t)\right|+\left|u_{y}(\mathbf{r}, t+\Delta t)-u_{y}(\mathbf{r}, t)\right|}{\sum_{\mathbf{r}}\left|u_{x}(\mathbf{r}, t)\right|+\left|u_{y}(\mathbf{r}, t)\right|}<T
$$

where $T$ is a tolerance set to $10^{-6}$. In figure 4 we show the results obtained with the collisional scheme. The time $t_{r}$ required to reach the steady state decreases at increasing values of $\tau$. The analytic solution of the Navier-Stokes equation for a single fluid subject to a Couette flow suggests a similar behaviour. For a single fluid it can be shown that the non-stationary part of the velocity profile behaves as a series of exponential terms $\exp \left(-\pi^{2} m^{2} \nu t / L^{2}\right)$ with $m$ an integer (Schlichting 1979). For this reason we have reported our results in a log-log scale, even if in this plot we do not observe a linear behaviour.

Next we consider the behaviour of the slip velocity. It is defined as the difference between the wall velocity and the fluid velocity along the wall itself and should be negligible. The slip velocity can be measured by the maximum relative error. At the top boundary it can be calculated as

$$
E_{m}=\max \left|\frac{u_{x}(i, L)-w_{x, t}}{w_{x, t}}\right| \quad i=1,2, \ldots, L
$$


where again $u_{x}(i, L)$ is the fluid velocity at the $i$-th site along the $x$ direction at the top boundary and $w_{x, t}=\gamma(L-1) / 2$ is the wall velocity. The results of this measure for different values of $\tau$ and for both schemes are presented in figure 5. For the collisional scheme $E_{m} \simeq 10^{-5}$ over the explored range $[0.7,20]$ of values of $\tau$ (figure 5 (a)). In the equilibrium scheme $E_{m}$ has a minimum value at $\tau=1$ and then increases with $\tau$ (figure 5 (b)) arriving to values of order 0.3. The conclusion is that the first scheme gives less convincing results and we have preferred to use the collisional scheme to run our simulations on phase separation.

\section{Simulations of phase separation}

We have studied the dynamics of phase separation on systems with size $L=256$, using the values written in section 2 for the parameters of the model and varying $\gamma$ in the range $[0.001,0.01]$ and $\tau$ in $[0.7,5]$, being mainly interested in the hydrodynamic regime at low viscosity.

Here we report the results obtained for $\tau=0.7$, which corresponds to a viscosity $\nu=$ 0.067, and $\gamma=0.005$. Similar results have been obtained for other values of parameters. A sequence of configurations at different values of the shear strain $\gamma t$ is shown in the left column of figure 6 . The first step of the time evolution is the formation of well defined interfaces. Then, for a while, domains grow isotropically as in the case without shear. At $\gamma t=0.4$, for example, the effects of the shear can be observed only near the moving walls due to the fact that at this time the linear profile of the velocity is not yet reached and the velocity is greater close to the walls (see figure 3). The deformation induced by the flow can be observed everywhere in the system starting from values $\gamma t \simeq 1$. Later on the domains 
become very stretched and progressively aligned with the flow direction as it can be clearly seen at $\gamma t=7$ and 11. It is also clear, by looking at these two configurations, that the system shows inhomogeneities and in particular domains with different thicknesses.

A quantitative analysis of the length scales present in the system can be done by considering the behaviour of the structure factor $C(\mathbf{k}, t)$. This quantity is the one of experimental interest being accessible through scattering techniques. It corresponds to the Fourier transform of the two-points correlation function and can be computed numerically as $\langle\varphi(\mathbf{k}, t) \varphi(-\mathbf{k}, t)>$, where $\varphi(\mathbf{k}, t)$ is the Fourier transform of the order parameter $\varphi(\mathbf{r}, t)$ and $\langle\ldots\rangle$ refers to an average over an ensemble of different initial realizations of the system. The wave vector $\mathbf{k}$ in the reciprocal lattice is given by $\mathbf{k}=\frac{2 \pi}{L}\left(n_{x} \mathbf{i}+n_{y} \mathbf{j}\right)$, where $\mathbf{i}$ and $\mathbf{j}$ are two unit vectors in the $k_{x}$ and $k_{y}$ directions, respectively, and $n_{x}$ and $n_{y}$ range from $-L / 2$ to $L / 2-1$. It could be useful to remind the behaviour of $C(\mathbf{k}, t)$ in the case without shear. Its shape is that of a volcano with a contour plot similar to that shown in figure 6 at $\gamma t=0.4$. The radius of the volcano defines the inverse of a characteristic length representing the average size of domains at a given time. As time goes by, the height of this volcano increases while its support shrinks towards the origin. This means that the characteristic length scale associated with the size of domains increases and the system is more and more ordered on that scale. The fact that the structure factor is circular reflects the isotropy of the system.

The shear affects in a significative way the evolution of the structure factor. At $\gamma t=1$ the shape of the volcano is deformed into an elliptical structure as it can be seen in the contour plot of figure 6. Moreover also the profile of the edge of the volcano is deformed and 
two maxima can be observed distributed around the main diagonal of the $k_{x}-k_{y}$ plane. In the further evolution the two broad maxima at $\gamma t=1$ become divided into two separate foils and on each foil two different peaks can be observed. This can be clearly seen at $\gamma t=7$. These two foils becomes closer and closer to the axis $k_{x}=0$ and increasingly more aligned along the $k_{y}$ axis. This pattern corresponds to interfaces very elongated in the direction of the flow as it appears in the corresponding pictures of configurations.

The presence of two peaks on each foil implies the existence of different length scales in the systems. Due to the symmetry $C(\mathbf{k}, t)=C(-\mathbf{k}, t)$ the two foils are perfectly symmetric and it is sufficient to consider only the two peaks of one foil. Therefore for each direction there are two relevant length scales. Focussing on what happens in the direction normal to the flow we arrive to the conclusion that there are domains with two characteristic thicknesses in the system. This better explains the inhomogeneities observed in the configurations at $\gamma t=7$ and 11

Also the dynamical evolution of the 4 -peaked structure factor is very peculiar. At $\gamma t=7$ the peaks which prevail are the ones with a larger value of $k_{y}$. This corresponds to a prevalence of thin domains in the system, as it can perhaps be observed by directly looking at the corresponding configuration. Later on the more isotropic peak with the smaller value of $k_{y}$, which has grown faster than the other, becomes higher. This can be observed in figure 6 at $\gamma t=11$ in correspondence of a larger abundance of thicker domains.

The physical picture of what happens in the system is the following. By stretching the interfaces the shear induces a stress in the system. This stress increases more and more producing a prevalence of thin domains. At a certain point the domains are broken by the 
flow in points where the stress is larger. This occurs in a cooperative way with a sequence of ruptures producing domains which are less stretched. Then the thicker domains, not yet broken by the flow, become more numerous and the more isotropic peak of $C(\mathbf{k}, t)$ dominates as at $\gamma t=11$. It was not possible to follow longer in our simulations the alternate predominance of the two peaks due to inevitable appearance of finite-size effects. Previous analytical results of Corberi et al. (1998) show that in the diffusive regime the alternate prevalence of the two peaks continues periodically on a logarithmic time scale. We cannot give results on this. Here we mention that in experiments with polymer solutions by Migler et al. (1996), after a shallow quench under the critical temperature, segregation is observed with 4-peaked structure factors similar to those of figure 6. Moreover, in previous experimental papers by Laufer et al. (1973) and Mani et al. (1991)-(1992) always on polymer solutions, the description of the dynamics of the network of domains is similar to that given above. We believe that our simulations, where for the first time the presence of two length scales is considered, help to understand these experimental facts.

We conclude this section giving the results of an explicit evaluation of the size of the domains. This measure is usually obtained from the momenta of the structure factor. Since our system is anisotropic we define the average size of domains in the flow direction as

$$
R_{x}(t)=\pi \frac{\int d \mathbf{k} C(\mathbf{k}, t)}{\int d \mathbf{k}\left|k_{x}\right| C(\mathbf{k}, t)}
$$

and analogously for $R_{y}(t)$, in the shear direction normal to the flow. They are one half of the wave length corresponding to the characteristic wave vector. In the case without shear lattice Boltzmann simulations with our parameters give $R_{x}=R_{y} \sim t^{2 / 3}$ (Osborn et al. 1995). $\alpha=2 / 3$ is the value of the growth exponent typical of the inertial regime which occurs at 
very low viscosity (Furukawa 1985). From our simulations we extract the behaviour shown in figure 7. As in all the other simulations with a shear flow (Yeomans 1999), it has not been possible to extract the value of the exponent $\alpha$ due to the relevance of finite-size effects ‥ Using an argument proposed by Corberi et al. (1999), based on a renormalization group approach (Bray 1990), one should expect in the present case, if dynamical scaling for the structure factor holds, that the growth in the shear direction obeys the same power law as in the case without shear, $R_{y}(t) \sim t^{2 / 3}$, while in the $x$-direction $R_{x}(t) \sim \gamma t R_{y}(t) \sim \gamma t^{5 / 3}$. This means that the growth exponent $\alpha_{x}$ in the flow direction is related to $\alpha_{y}$, the growth exponent in the shear direction, by the relation $\alpha_{x}=\alpha_{y}+1$, where the extra contribution 1 with respect to the unsheared case comes from the convective term in the convectiondiffusion equation (18). Our runs, performed on an Alpha Workstation XP1000 with 1 GB of RAM, do not allow to check these predictions. The results of figure 7 can be more easily related to the above discussed dynamical behaviour of the structure factor with the alternate dominance of the two peaks which corresponds to the oscillatory pattern of $R_{x}$ and $R_{y}$. We also observe that these oscillations make more difficult a possible evaluation of the growth exponents.

\section{Conclusions}

We studied phase separation in sheared binary fluid mixtures at low viscosity. We introduced proper boundary conditions to impose the shear flow on the system. These boundary conditions strictly conserve mass and momentum and do not introduce any appreciable slip

\footnotetext{
${ }^{3}$ An analytical determination of the exponent $\alpha$ is only available for the diffusive regime (Corberi et al. (1998), Rapapa \& Bray (1999)). It is found that $\alpha=4 / 3$ in the flow direction, and $\alpha=1 / 3$ in the other directions as in the case without shear.
} 
velocity. Our main results show that the network of domains is characterized by the existence of two typical length scales for each direction. Indeed, domains with different thickness are clearly visible in the simulations. Structure factors with four peaks confirm these observations. During the time evolution there is an alternate dominance of two of these peaks over the other couple. This corresponds to a larger abundance first of thin and later of thicker domains. Simulations on much larger scales are needed to establish if the oscillations of the peaks only characterize an initial transient or if they continue indefinitely, as it is suggested by the results of Corberi et al. (1998) valid for the diffusive regime at infinite viscosity.

\section{Ackowledgements}

We thank A. Bonfiglioli for useful preliminary discussions. G.G. acknowledges support by MURST (PRIN97). 


\section{Appendix}

A suitable choice of the coefficients in the expansions (14)-(15) consistent with the conditions (10)- 1 (13) is

$$
\begin{gathered}
A_{0}=n-20 A_{I I}, \quad A_{I}=4 A_{I I}, \quad A_{I I}=\frac{P_{x x}+P_{y y}}{24} \\
B_{I}=4 B_{I I}, \quad B_{I I}=\frac{n}{12} \\
C_{0}=-\frac{2 n}{3}, \quad C_{I}=4 C_{I I}, \quad C_{I I}=-\frac{n}{24} \\
D_{I}=4 D_{I I}, \quad D_{I I}=\frac{n}{8} \\
G_{I, \alpha \beta}=4 G_{I I, \alpha \beta}, G_{I I, x x}=-G_{I I, y y}=\frac{P_{x x}-P_{y y}}{16}, \quad G_{I I, x y}=-G_{I I, y x}=\frac{P_{x y}}{8} \\
H_{0}=\varphi-20 H_{I I}, \quad H_{I}=4 H_{I I}, \quad H_{I I}=\frac{\Gamma \Delta \mu}{12} \\
K_{I}=4 K_{I I}, \quad K_{I I}=\frac{\varphi}{12} \\
J_{0}=-\frac{2 \varphi}{3}, \quad J_{I}=4 J_{I I}, \quad J_{I I}=-\frac{\varphi}{24} \\
Q_{I}=4 Q_{I I}, \quad Q_{I I}=\frac{\varphi}{8}
\end{gathered}
$$




\section{References}

Bhatnagar, P., Gross, E. P. \& Krook, M. K. 1954 Phys. Rev. 94, 511.

Bray, A. J. 1990 Phys. Rev. B 41, 6724.

Bray, A. J. 1994 Adv. Phys. 43, 357.

Cates, M. E., Kendon, V. M., Bladon, P. \& Desplat, J. C. 1999 Faraday Discussions 112, 1.

Chapman, S. \& Cowling, T. 1970 The Mathematical Theory of Non-uniform Gases (Cambridge University Press, Cambridge).

Chen, H., Chen, S. \& Matthaeus, W. 1992 Phys. Rev. A 45, R5339.

Chen, S. \& Doolen, G. D. 1998 Annu. Rev. Fluid Mech. 30, 329.

Corberi, F., Gonnella, G. \& Lamura, A. 1998 Phys. Rev. Lett. 81, 3852.

Corberi, F., Gonnella, G. \& Lamura, A. 1999 Phys. Rev. Lett. 83, 4057.

Cornubert, R., d'Humieres, D. \& Levermore, D. 1991 Physica D 47, 241.

Evans, R. 1979 Adv. Phys. 28, 143.

Furukawa, H. 1985 Adv. Phys. 34, 703.

Gonnella, G., Orlandini, E. \& Yeomans, J. M. 1997 Phys. Rev. Lett. 78, 1695.

Gunton, J. D., San Miguel, M. \& Sahni, P. S. 1983 in Phase Transitions and Critical Phenomena, edited by C. Domb, J. L. Lebowitz (Academic, New York).

Hashimoto, T., Matsuzaka, K., Moses, E. \& Onuki, A. 1995 Phys. Rev. Lett. 74, 126.

He, X., Zou, Q., Luo, L. S. \& Dembo, M. 1997 J. Stat. Phys. 87, 115.

Inamuro, T., Yoshino, M. \& Ogino, F. 1995 Phys. Fluids 7, 2928.

Kendon, V. M., Desplat, J. C., Bladon, P. \& Cates, M. E. 1999 Phys. Rev. Lett. 83, 576. 
Ladd, A. J. 1994 J. Fluid Mech. 271, 285.

Landau, L. D. \& Lifshitz, E. M. 1959 Fluid Mechanics (Pergamon Press, Oxford).

Laufer, Z., Jalink, H. L. \& Staverman, A. J. 1973 Journal of Polymer science 11, 3005.

Lavallée, P., Boon, J. \& Noullez. A. 1991 Physica D 47, 233.

Lees, A. W. \& Edwards, S. F. 1972 J. Phys. C 5, 1921.

Mani, S., Malone, M. F., Winter, H. H., Halary, J. L. \& Monnerie, L. 1991 Macromolecules 24, 5451 .

Mani, S., Malone, M. F. \& Winter, H. H. 1992 Macromolecules 25, 5671.

Migler, K., Liu, C. \& Pine, D. J. 1996 Macromolecules 29, 1422.

Noble, D. R., Chen, S., Georgiadis, J. G. \& Buckius, R. O. 1995 Phys. Fluids 7, 203.

Ohta, T., Nozaki, H. \& Doi, M. 1990 Phys. Lett. A 145, 304; J. Chem. Phys. 93, 2664.

Olson, J. F. \& Rothman, D. H. 1995 J. Stat. Phys. 81, 199.

Onuki, A. 1997 J. Phys. Condens. Matter 9, 6119.

Orlandini, E., Swift, M. R. \& Yeomans, J. M. 1995 Europhys. Lett. 32, 463.

Osborn, W. R., Orlandini, E., Swift, M. R., Yeomans, J. M. \& Banavar, J. R. 1995 Phys. Rev. Lett. 75, 4031.

Padilla, P. \& Toxvaerd, S. 1997 J. Chem. Phys. 106, 2342.

Rapapa, N. P. \& Bray, A. J. 1999 Phys. Rev. Lett. 83, 3856.

Reichl, L. E. 1980 A Modern Course in Statistical Physics (Arnold, London).

Rothman, D. H. 1991 Europhys. Lett. 14, 337.

Rowlinson, J. S. \& Widom, B. 1982 Molecular Theory of Capillarity (Clarendon Press, Oxford). 
Schlichting, H. 1979 Boundary Layer Theory (Mc Graw Hill series in Mechanical Engineering).

Shou, Z. \& Chakrabarti, A. 2000 Phys. Rev. E 61, R2200.

Swift, M. R., Orlandini, E., Osborn, W. R. \& Yeomans, J. M. 1996 Phys. Rev. E 54, 5041.

Wagner, A. J. \& Yeomans, J. M. 1999 Phys. Rev. E 59, 4366.

Wu, Y. N., Skrdla, H., Lookman, T. \& Chen, S. Y. 1997 Physica A 239, 428.

Yang, A. J. M., Fleming, P. D. \& Gibbs, J. H. 1976 J. Chem. Phys. 64, 3732.

Yeomans, J. M. 1999 preprint OUTP-99-06S, Ann. Rev. Comp. Physics, ed. D. Stauffer, in press.

Zou, Q. \& He, X. 1997 Phys. Fluids 9, 1591. 


\section{Figure Captions}

Figure 1: One plaquette of the lattice used in our model with the velocity vectors $\mathbf{e}_{i}$ $(\Delta t=1)$.

Figure 2: Plots of the total fluid density $n$ (a) and of the density difference $\varphi$ (b) along a vertical section at $t=32$, using the collisional scheme. Simulations were run with $L=128, \gamma=0.01$ and $\tau=1$.

Figure 3: Plot of the $x$-component of the fluid velocity as a function of the $y$ coordinate at consecutive times: $(\triangle) t=2,(\circ) t=8,(\star) t=16,(\bullet) t=32$ for the collisional sheme of shear boundary conditions.

Figure 4: The time $t_{r}$ required to reach the steady state is shown as a function of the relaxation parameter $\tau$, using the collisional scheme.

Figure 5: The maximum relative error $E_{m}$ in the velocities at the top boundary is reported for the collisional (a) and the equilibrium (b) scheme as a function of $\tau$. Measures were taken at the times shown in figure 4.

Figure 6: Configurations of the system are shown in the left column at different values of the shear strain $\gamma t$. In the right column the structure factor is contour-plotted at the same values of $\gamma t$.

Figure 7: Evolution of the average domain size in the shear (lower curve) and flow (upper curve) directions. The slope of $R_{x}$ is 1.1. 


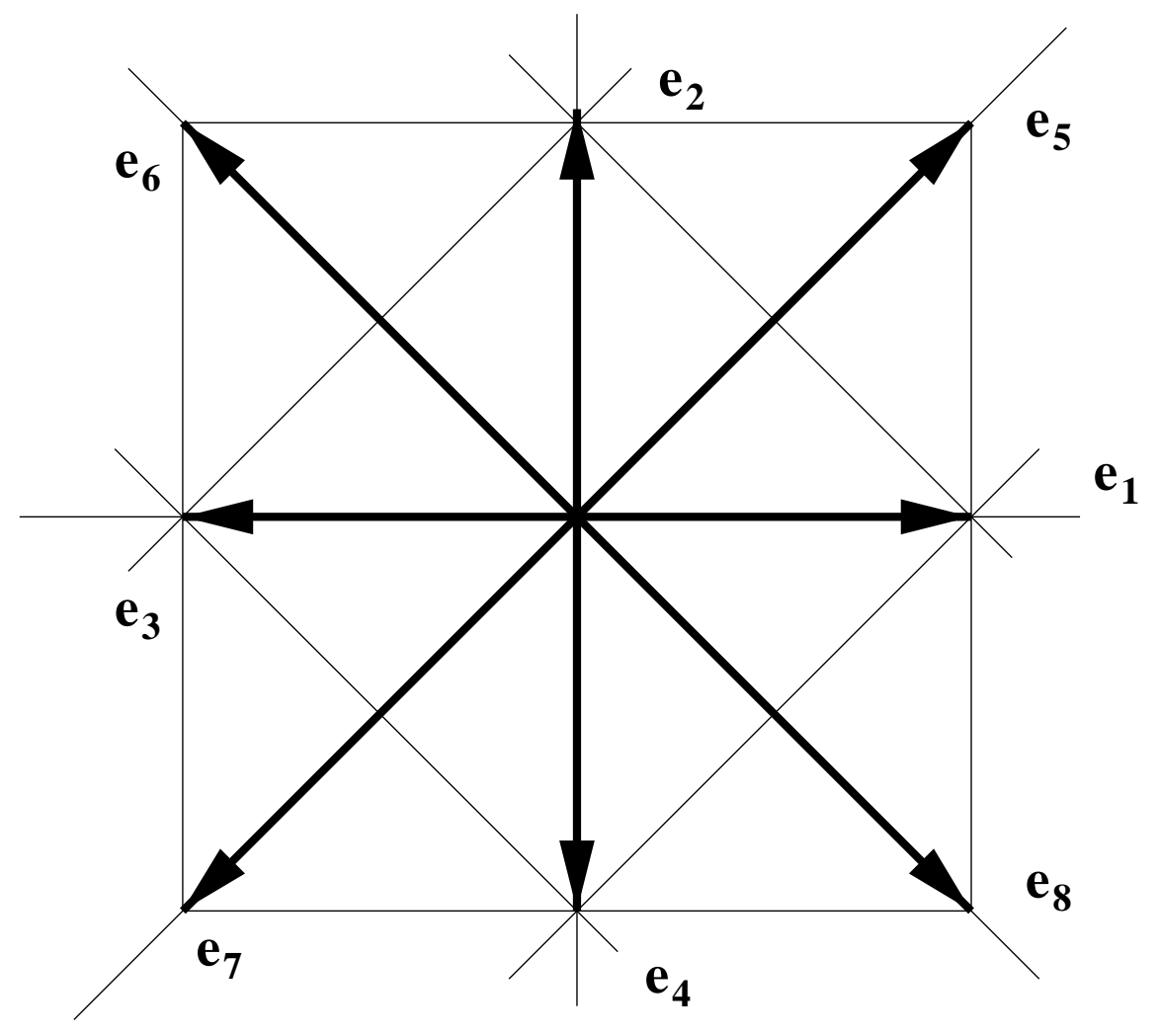

Figure 1 


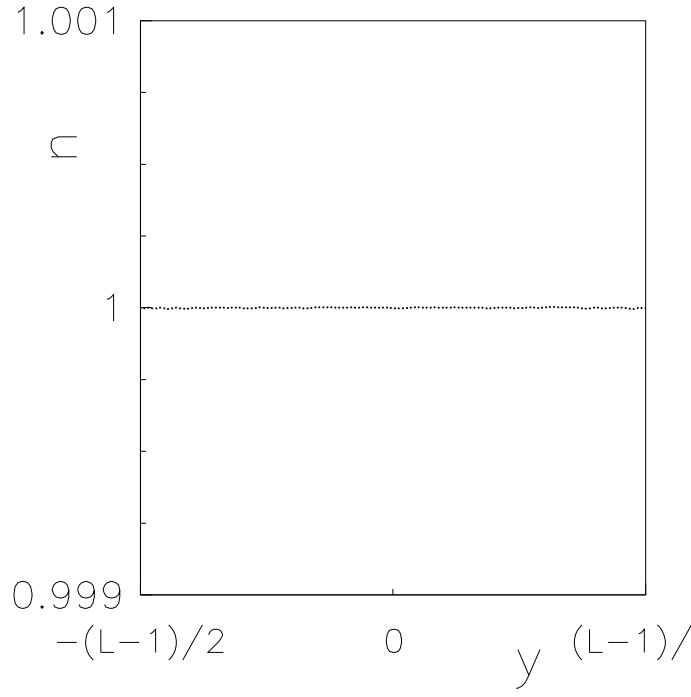

(a)

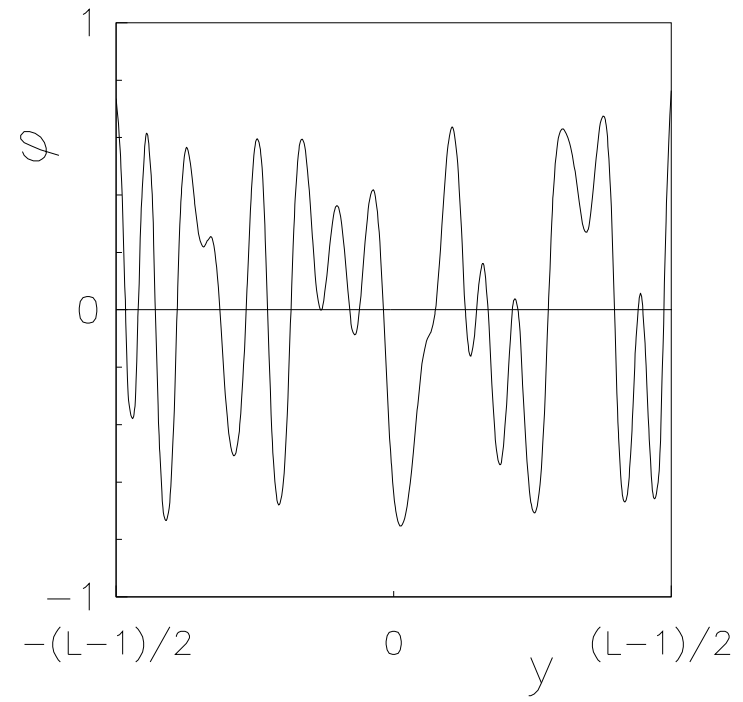

(b)

Figure 2 


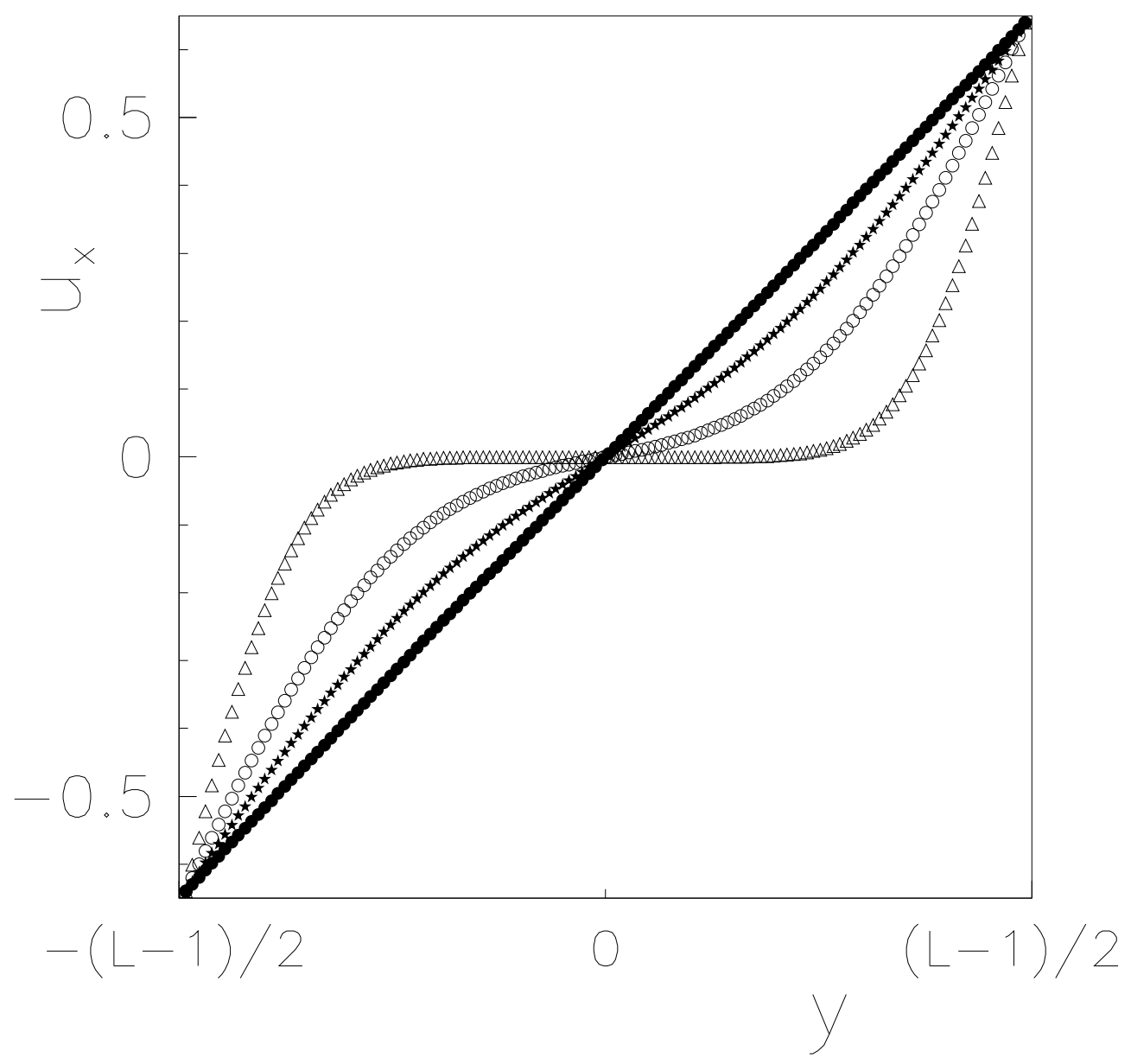

Figure 3 


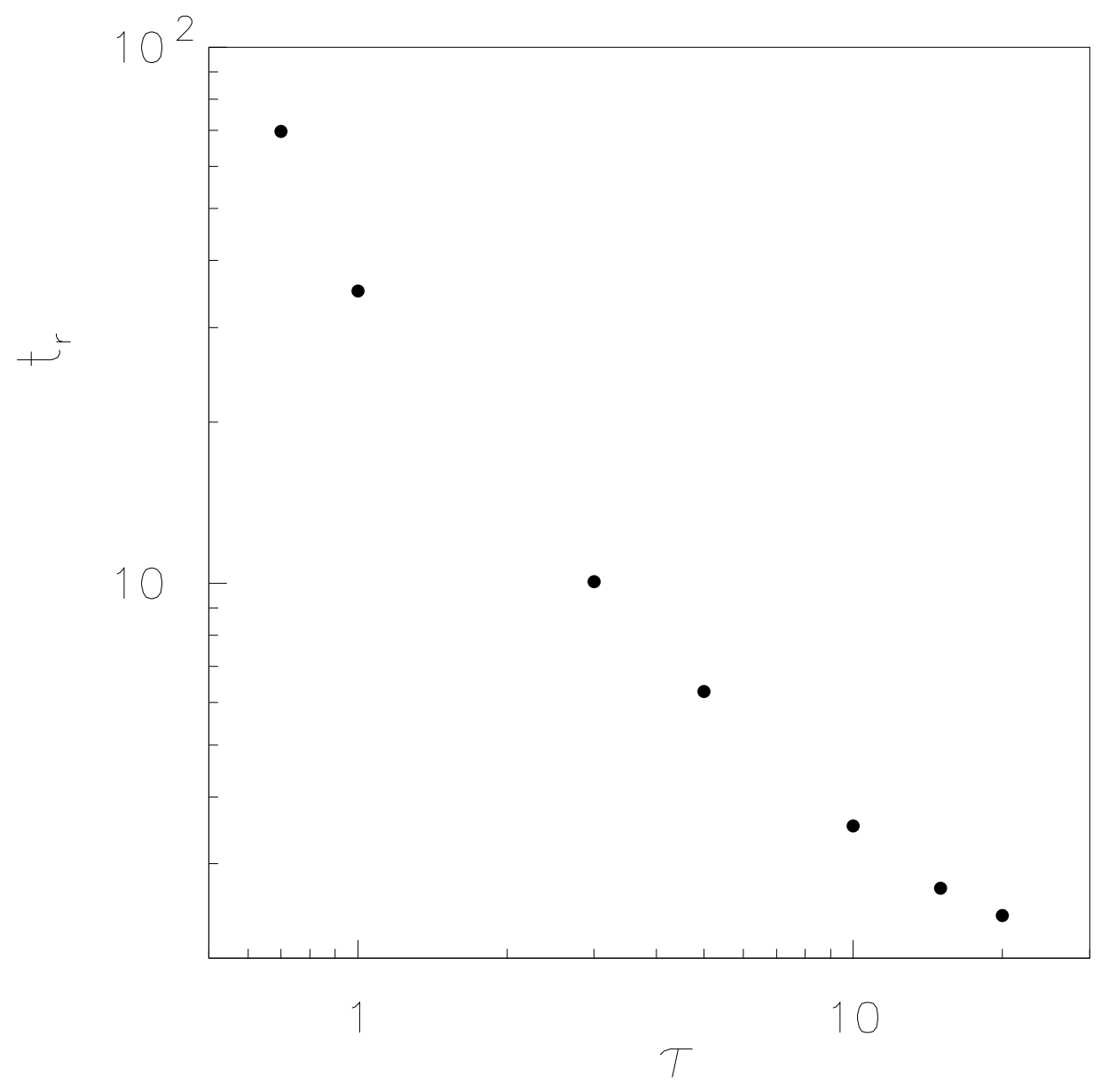

Figure 4 


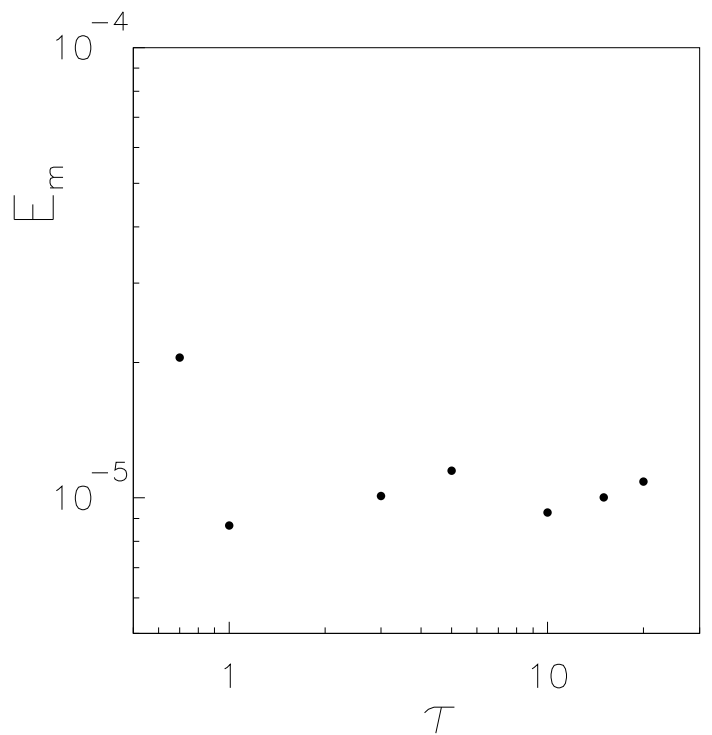

(a)

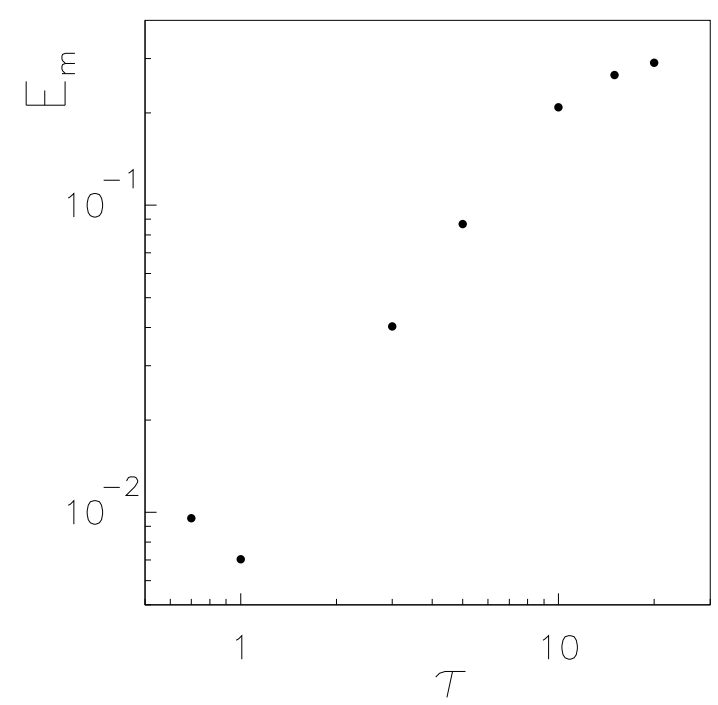

(b)

Figure 5 


\section{$\gamma t=0.4$}

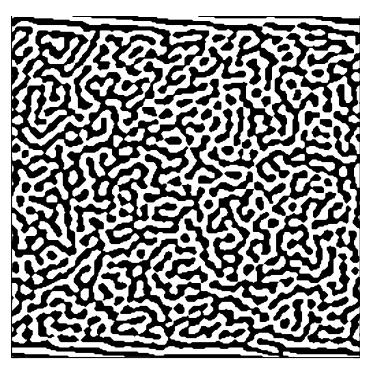

$\gamma t=1$

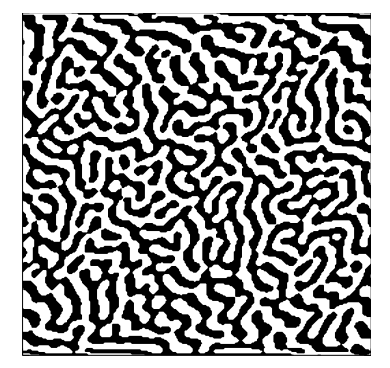

$\gamma t=7$

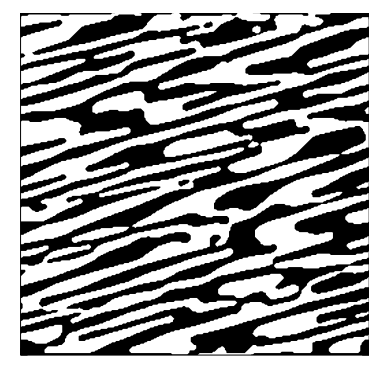

$\gamma t=11$

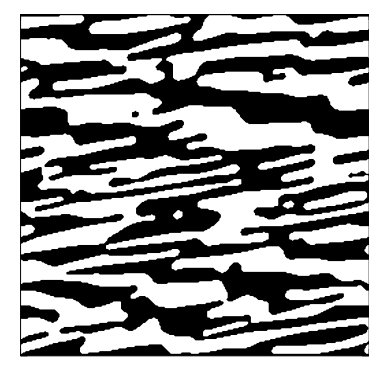

$\gamma t=0.4$

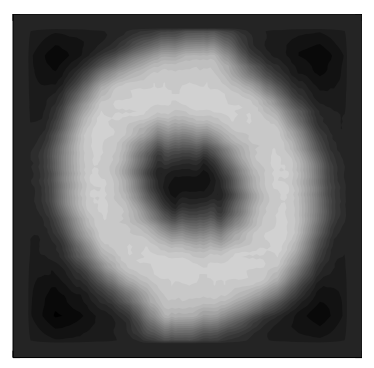

$\gamma t=1$

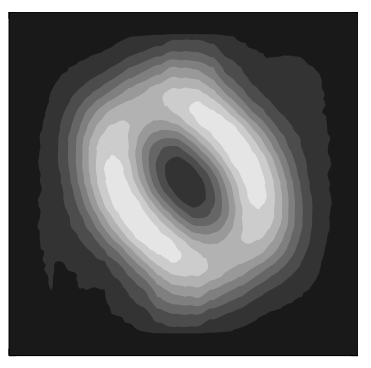

$\gamma t=7$

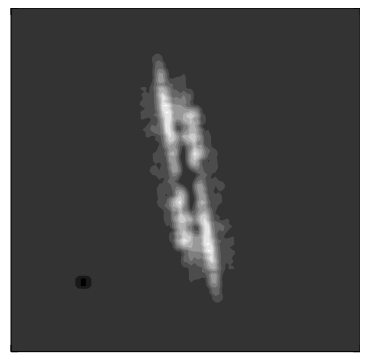

$\gamma t=11$

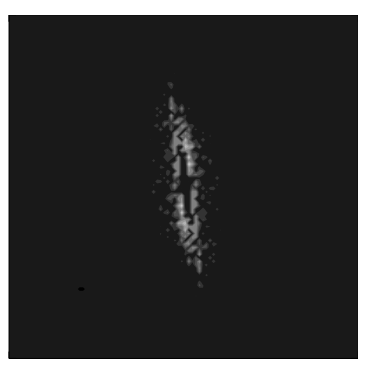

Figure 6 


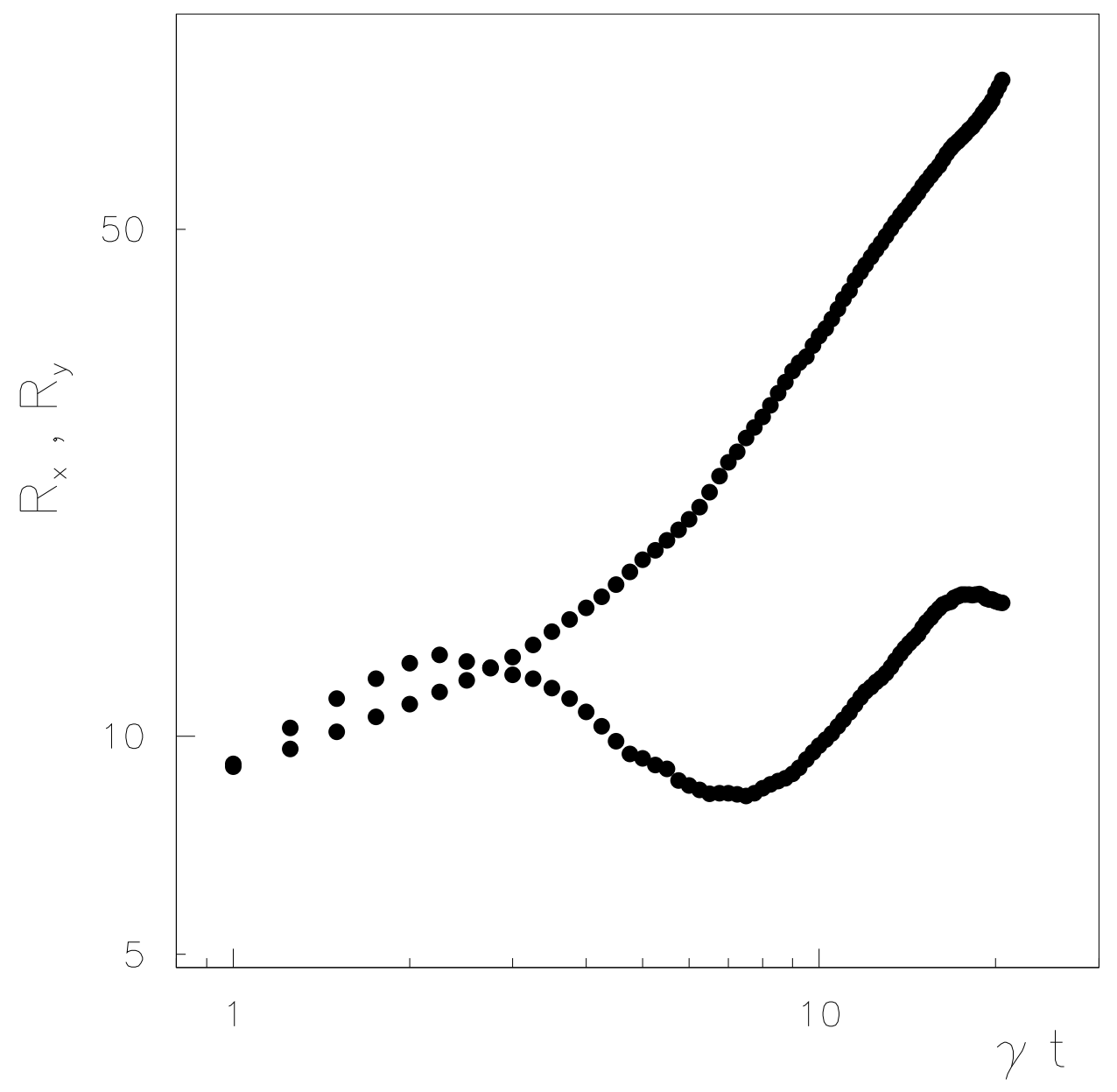

Figure 7 